\title{
Süchte, "Verhaltenssüchte» und die neue «Sucht-Sucht»
}

\author{
Iver Hand \\ "Spielerprojekt Hamburg», MVZ Verhaltenstherapie Falkenried (bis 2008 Uniklinik Hamburg), Hamburg, Deutschland
}

\begin{abstract}
Prolog
In den letzten 10 Jahren ist es in Teilen der Fachpresse und in den öffentlichen Medien zu einem inflationären Gebrauch der Suchtdiagnose gekommen - vor allem auch durch deren Ausweitung auf die «Verhaltenssüchte». Das hat im Versorgungssystem zu deutlichen Verwerfungen geführt. Aktuell empfehlen prominente Vertreter des Suchtbereichs offenbar eine völlige Löschung der Diagnose «Sucht». Nicht nur die Patienten, sondern auch wir Verhaltenstherapeuten waren und sind von diesen Vorgängen direkt betroffen. Vielleicht kann dieser Beitrag einem besseren Verständnis der gelaufenen - und damit auch der zukünftigen - Entwicklung dienen.
\end{abstract}

\section{Von der «Sucht-Sucht» zur «Sucht»-Abstinenz?}

In der aktuellen Ausgabe der von der Deutschen Gesellschaft für Suchtforschung und Suchttherapie herausgegebenen Fachzeitschrift «SUCHT» plädieren 2 Chefredakteure im Editorial für die komplette Abschaffung des «Sucht»-Begriffs [Bühringer und Rumpf, 2018]. Diese solle in allen entsprechenden Einrichtungen sowie «in allen Informationsmedien und in der Öffentlichkeitsarbeit» umgesetzt werden. Als Hauptgründe führen Sie auf:

- Begriffe wie «Sucht»-Kranke, -Hilfe oder -Therapie seien stigmatisiert und würden zu einer zu geringen Inanspruchnahme der gegenwärtigen Versorgungsstruktur führen; besonders Personen in frühen Gefährdungsstadien würden dadurch abgeschreckt;

- die von der Suchtdiagnose abgeleitete und in der Öffentlichkeitsarbeit immer noch dominierende "Abstinenzforderung» sei besonders hinderlich für die Präventionsarbeit und müsse durch das Ziel ersetzt werden, «Konsumverhalten so zu steuern, dass der Genuss maximiert und die Risiken im Lebensalltag minimiert werden»;

Weitere Informationen finden sich unter: $w w w$.hand.vt-falkenried.de; www.spielerprojektprof-hand.vt-falkenried.de.
- Suchtdiagnosen seien im Zeitverlauf nicht stabil (eigene Anmerkung: Die klassische Suchtdiagnose impliziert allerdings das Gegenteil).

Diese Argumente wurden außerhalb der Suchteinrichtungen mindestens in den letzten 4 Jahrzehnten national und international immer wieder publiziert. Daher stellt sich die Frage, ob in dieser Zeit durch die massive Propagierung der Suchtdiagnose für Verhaltensexzesse wirksamere Angebote zum Schaden etlicher Hilfsbedürftiger nicht gefördert bzw. behindert wurden. Gründe hierfür könnten mangelndes psychotherapeutisch-psychopathologisches Wissen mancher Meinungsbildner, macht- und verbandspolitische Interessen sowie - «last but not least» - vielleicht auch politische Interessen sein.

Im Folgenden soll versucht werden, diese Vermutungen am Beispiel der «Verhaltenssüchte», die vor allem mit der «Glücksspielsucht» begründet wurden, zu untermauern.

\section{Die Suchtdiagnose: Ein Chamäleon und seine (Be-)Nutzer}

\section{Die Suchtdiagnose als sozialpolitisches Instrument}

Anerkennung von Problem- und Risikoverhalten als Krankheit:

Die Einführung der «Sucht»-Diagnose sollte ursprünglich Folgendes bewirken: die Anerkennung als Krankheit, mit entsprechenden Versicherungsleistungen; einen Schutz vor dem Makel, als willensschwach zu gelten und «selbst schuld» am Problemverhalten zu sein; eine juristische Entlassung bei delinquentem Verhalten im Zusammenhang mit dem Problemverhalten. Für diese sozialpolitischen Ziele wäre die Suchtdiagnose heute verzichtbar.

\section{Steuerung des Geldflusses in der Krankenversorgung}

Das Urteil des Bundesverfassungsgerichts vom März 2006, demzufolge Glücksspiel zur «Sucht» werden kann, beinhaltete, dass alle Glücksspielanbieter fortan die Prävention von, Therapie bei und Forschung zu Glücksspielsucht finanziell nachhaltig för-

\section{KARGER}

() 2018 S. Karger GmbH, Freiburg

Fax +497614520714
Prof. Dr. med. Iver Hand

MVZ Falkenried

Falkenried 7

20251 Hamburg, Deutschland

hand@vt-falkenried.de 
dern mussten, um die entsprechende Konzession zu behalten oder $\mathrm{zu}$ erwerben. Aus dem daraus resultierenden ersten Glücksspielstaatsvertrag (Januar 2008) ist der «Spielsucht»-Begriff in den öffentlichen Medien und der Fachpresse massiv propagiert worden.

Dies führte hinsichtlich der «Spielsucht» zwar erfreulicherweise $\mathrm{zu}$ erheblichen Geldzuwendungen vonseiten der privaten und staatlichen (Bundesländer) Glücksspielanbieter für Forschung, Prävention und Therapie - aber leider fast ausschließlich für die Leitdiagnose «Sucht». So wurden auch auf der Therapieseite weit überwiegend Suchteinrichtungen gefördert, was teilweise nur einen mäßigen Erfolg für die betroffenen Spieler nach sich zog, z.B. durch mangelnde Inanspruchnahme der Suchtberatung, hohe Abbruchquoten nach dem Erstkontakt und zusätzliche hohe Abbruchquoten innerhalb der ersten 5 Sitzungen (Details z.B. in Hand und Kegat [2013]). Die national und international vorliegende Literatur zur Wirksamkeit der kognitiven Verhaltenstherapie (KVT) wurde in diesem Prozess weitgehend ausgeblendet.

Spätestens seit 2014 wurde die «Sucht»-Diagnose dann von vielen einflussreichen Autoren aus dem Bereich der Psychiatrie und Psychotherapie auf etliche weitere Verhaltensexzesse in Richtung «Verhaltenssüchte» ausgeweitet (für einen Überblick siehe Mann [2013, 2014] und Bilke-Hentsch et al [2014]). Als Therapieform für diese «neuen Süchte» wird in diesen Publikationen aber nicht Suchtberatung sondern überwiegend KVT empfohlen - und das ohne Bezugnahme auf die diesbezüglichen deutschsprachigen Publikationen seit Mitte der 1980er Jahre.

Dies wirft für das deutsche Versorgungssystem natürlich die Fragen auf, weshalb die eine Sucht in der Suchtberatung und die andere mit KVT zu behandeln ist (siehe unten) und worin der Unterschied zwischen den beiden Süchten und den beiden Betreuungsangeboten besteht.

\section{Die Süchte zu den Suchteinrichtungen?}

In Deutschland galt Jahrzehnte lang, dass Süchte in Suchteinrichtungen mit suchtspezifischen Maßnahmen zu betreuen und die Kosten dafür mit einem speziellen Budget zu finanzieren sind. Psychiatrische und psychotherapeutische Therapien wurden aus einem anderen Topf finanziert. Nach der aktuellen Psychotherapie-Richtlinie der Kassenärztlichen Bundesvereinigung [KBV, 2017] gilt noch, dass ein Suchtpatient, wenn er eine primäre Psychotherapie erhält, bis zur 10. Sitzung eine Abstinenz erreicht haben muss und andernfalls zwecks Entzugs eine Suchteinrichtung zwischenzuschalten ist. Diese Regel macht schon bei stoffgebundenen Süchten nicht immer Sinn. Für «Verhaltenssüchte» gilt sie nicht, denn die Diagnose hat bisher keinen Eingang in die Psychotherapie-Richtlinie gefunden.

$\mathrm{Zu}$ diesem Themenkomplex eine kurze historische Anmerkung: Nachdem erste Publikationen aus Deutschlands erstem Therapieforschungsprojekt zu pathologischem Glücksspielen (seit 1984 an der Verhaltenstherapie-Ambulanz der Psychiatrischen Uniklinik in Hamburg-Eppendorf) veröffentlicht waren, lud die Katholische Sozialethische Akademie Mitarbeiter des Projekts ein, in ihren Einrichtungen Seminare für deren MitarbeiterInnen und Interessierte aus anderen Suchteinrichtungen zu geben. Diese liefen sehr erfolg- reich, bis sie mit einem Rundschreiben des Deutschen Caritasverbands Freiburg (April 1988) indirekt untersagt wurden - mit dem Hinweis, Spielsüchtige müssten vor allem in den Suchteinrichtungen der Caritas betreut werden.

\section{Die Suchtdiagnose zur Selbstexkulpierung des Staats?}

In den 1970er Jahren regulierten die meisten Staaten den Glücksspielmarkt noch recht restriktiv. Da die Nachfrage der Bürger nach Glücksspielangeboten in vielen Ländern erheblich zugenommen hat, wurden diese inzwischen selbst in Staaten wie China und Singapur legalisiert. Hierzu sei eine «spielerische» Hypothese gewagt: Die Verlockung, Bürger über die Besteuerung von Glücksspiel zu freiwilligen und erheblichen zusätzlichen Steuerzahlungen zu motivieren, wurde einfach zu groß. Daher wurde der Staat auch in Deutschland durch die Kontrolle über die Lizensierung und die Besteuerung z.B. von Lotto/Toto, Casinos und Spielhallen indirekt zum erfolgreichen Glücksspielunternehmer. Da Glücksspielen aber nach der Meinung vieler Bürger grundsätzlich etwas Schlechtes ist, benötigten Staaten vielleicht eine «Entschuld(ig)ung» für das Elend gescheiterter Spieler. Wenn Spiel-«Sucht» sich nur auf dem Boden einer biologischen Veranlagung entwickelt, wäre der Staat dafür vielleicht nicht primär verantwortlich, sondern er trüge lediglich die Verantwortung für den Schutz der Gefährdeten. Für die Sicherstellung dieses Schutzes möchte er eigentlich ein Angebotsmonopol für Glücksspiele - mit dem angenehmen Nebeneffekt, dass seine Einnahmen dadurch noch weiter steigen würden. Kaum ein Staat kümmert sich aber adäquat um das mit weitem Abstand größte und gefährlichste Casino der Welt: die Börse.

Kriterien der Suchtdiagnose bei Verhaltensexzessen: Verhaltensanalytische Reformulierungen

Als Beleg für das Vorliegen einer Sucht werden immer wieder die folgenden Kernmerkmale angeführt, denen hier jeweils eine verhaltensanalytisch-psychopathologische Interpretation entgegengestellt wird:

Kontrollverlust über das eigene Konsumverhalten

Der «Kontrolle»-Begriff enthält implizit den Ruf nach Fremdkontrolle, wenn Selbstkontrolle nicht mehr funktioniert. Wenige Menschen sind jedoch bereit, Fremdkontrolle längerfristig zu akzeptieren. Das Ziel einer strategisch-systemisch ausgerichteten VT ist nicht, ein eigenes Verhalten oder das Verlangen danach zu kontrollieren, sondern die Handlungsmotivation dafür zu erkennen und mit Alternativen zu experimentieren. So bleibt die Autonomie des Patienten gewahrt, und eine verbindliche Therapiezielsetzung wird gefördert.

\section{Krankheit des Willens}

Die «Krankheit des Willens» wird in mehreren Varianten angeführt, aber nicht operationalisiert. Verhaltensanalytisch seien dazu die folgenden Überlegungen genannt:

- Unfreiheit des Willens: Der Wille des Spielers, zu spielen, ist grundsätzlich nicht unfreier als sein Wille, etwas anderes zu tun. Warum soll er bezüglich des Ersteren entmündigt werden? 
Eine vorurteilsfreiere Frage könnte lauten: «Warum will ein Mensch unbedingt das, was er nach dem Willen seines Umfelds nicht wollen sollte?» Auch diese Frage führt wieder zur Motivationsanalyse.

- Unwiderstehliches Verlangen (Craving) bei «Entzugssymptomen»: Als typische Spielsucht-Entzugssymptome werden 2 Symptomgruppen angeführt: 1) Unlust, Reizbarkeit, Schlafstörungen und Alpträume; 2) Schweißausbrüche, Zittern, motorische Unruhe und innere Unruhe. Für den erfahrenen Kliniker sind dies aber Symptome von Depression und Angst.

- Unfähigkeit, der Versuchung zu widerstehen: Auch dieser Begriff wird nicht operationalisiert, ist aber oft über eine eingehende individuelle und gegebenenfalls systemische Motivationsanalyse inhaltlich zu präzisieren.

Die Unfähigkeit, positive Gefühle gegenüber dem Suchtstoff zu unterdrücken

Dieses Konzept wurde ebenfalls auf Verhaltenssüchte übertragen. Es drückt die Annahme aus, dass exzessives Verhalten aus früher und anhaltender positiver Verstärkung resultiert. Für die meisten therapiesuchenden Spieler steht jedoch die Vermeidung negativer Gedanken und Gefühle mithilfe des Spielens (negative Verstärkung) deutlich im Vordergrund - mit entsprechenden therapeutischen Konsequenzen [Hand, 2004].

\section{Chronischer Verlauf}

Epidemiologische Studien mit pathologischen Glücksspielern haben klar gezeigt, dass ein wirklich chronischer Verlauf die Ausnahme und nicht die Regel ist. Bei diesen Ausnahmen ist dann oftmals nicht die Sucht chronisch, sondern die zugrundeliegende Erkrankung.

Chronischer Verlauf, der nur durch Medikamente behoben werden kann

Es mag auch solche phänotypischen Verläufe geben, wobei dann jeweils zu klären wäre, ob die Sucht oder eine zugrundeliegende psychische Störung zu behandeln sind.

\section{Chronischer Verlauf trotz längerer Abstinenzzeiten}

Warum formulieren wir nicht: chronische Gesundheit mit gelegentlichen Rückfällen in ein Krankheitsverhalten, die dann als «Vorfälle» interpretiert werden. Diese sind Anlass, innezuhalten, und, allein oder mit therapeutischer Hilfe, zu eruieren, welche neuen «Fehler in der Lebensführung» dazu geführt haben könnten. Für Betroffene kann es gravierend unterschiedliche Auswirkungen haben, ob der Therapeut das eine oder das andere Modell vertritt: biologisch bedingte Hilflosigkeit oder erlernte CopingKompetenz.

\section{Verhaltenstherapeutische Empfehlungen für Verhaltensexzesse}

Unter den Diagnosen «problematisches», «pathologisches» oder «süchtiges Glücksspielen» werden sehr heterogene Gruppen von Betroffenen zusammengefasst, bei denen sich die Indikation für spezifische Therapiemaßnahmen erst aus der individuellen Diagnostik, und nicht aus der Symptomdiagnose ergibt. Bei Verhaltensexzessen, insbesondere beim exzessiven Glücksspielen, kommt der frühen Klärung der Motivation zur Verhaltensänderung aus folgenden Gründen besondere Bedeutung zu:

Glücksspieler wollen grundsätzlich spielen. Viele pathologische Glücksspieler wollen spielen, weil es sie emotional-kognitiv entlastet - im Sinne einer negativen Verstärkung bzw. als «Versuch einer Selbstmedikation». Daraus ergibt sich der implizite Zweifel des Betroffenen: «Hat der Therapeut wirklich etwas Besseres zu bieten?» Für die Klärung dieser Frage empfahl schon Kanfer: «Joint the client where he is». Doch dann stellt sich die entscheidende Frage: «... but, where is he?» [Hand, 2008]. Damit landen auch wir Verhaltenstherapeuten wieder bei der individuellen und systemischen Analyse der Motivation zu einer Verhaltensänderung. Erst dieser Kernbereich der Diagnostik bei Verhaltensexzessen ermöglicht dann - zusammen mit einer kausal begründeten Hierarchisierung vorhandener Multisymptomatiken - die Entwicklung einer (Be-) Handlungsstrategie, in deren Rahmen entschieden werden kann, welche VT-Technik wann zur Anwendung kommen sollte. Für die Therapeut-Patient-Beziehung kann es hilfreich sein, die Interaktion zeitweise spielerisch-spannend oder auch kurzzeitig provokativ zu gestalten.

\section{Praxisbezogene Empfehlungen für eine Kooperation zwischen Verhaltenstherapie und Suchtberatung}

An dieser Stelle werden auf der Basis der real existierenden Versorgungssysteme für exzessive Glücksspieler - als pars pro toto Vorschläge für eine zügige Verbesserung der Versorgungssituation unterbreitet. Deren Realisierung setzt «nur» voraus, dass ideologische Barrieren zwischen den Versorgergruppen abgebaut werden und den Betroffenen vonseiten der Finanzierer bei klar indiziertem Bedarf diagnoseunabhängig ein Wechsel zwischen den Einrichtungen ermöglicht wird. Dann könnten sich die im folgenden Abschnitt aufgeführten Indikationen ergeben.

\section{Die Vorteile von auch für Verhaltensexzesse spezialisierter Verhaltenstherapie:}

- eine initial psychopathologische Differentialdiagnostik zur Identifikation von dem Verhaltensexzess möglicherweise zugrundeliegenden psychischen Störungen;

- bei Mehrfachstörungen (Komorbidität) deren Hierarchisierung nach einem therapieleitenden, übergeordneten Störungsmodell;

- eine schwerpunktmäßige Behandlung der identifizierten Hauptstörung (Ursachentherapie);

- eine symptomspezifische VT z.B. bei Spielern mit primär positiver Verstärkung des Symptomverhaltens;

- eine relativ hohe initiale Akzeptanz des Therapieangebots in der Bevölkerung. 
Die Vorteile von auch auf Spielerberatung spezialisierter (!) Suchtberatung:

- die häufige Verfügbarkeit einer sofortigen Krisenintervention;

- eine kurze Wartezeit für eine Erstberatung, auch von Angehörigen (einschließlich Vermittlung von Schuldnerberatung);

- seit einigen Jahren gute Beratungsrichtlinien für eine zumindest vorübergehende Reduktion des Spielverhaltens;

- eine Betreuung von Spielern, die (noch) keine Zusammenhänge zwischen ihrem Spielverhalten und anderen überfordernden Problemen in der Lebensführung erkennen (wollen/können?);

- bei einer Abstinenz als Therapieziel des Spielers: mancherorts ein gutes, stützendes Umfeld durch kooperierende Selbsthilfegruppen (die «Gamblers Anonymous» sind allerdings deutlich weniger erfolgreich als die «Anonymen Alkoholiker»).

Die meisten VT-Praxen können, strukturbedingt, auf eine krisenhafte Eskalation von Verhaltensexzessen nicht mit einem sofortigen Therapieangebot reagieren. Sie könnten aber über die Notfallsprechstunde die Kooperation mit einer Suchtberatungsstelle einleiten. Gemeinsame Fallkonferenzen könnten das gemeinsame Wissen steigern und das gegenseitige Vertrauen fördern. In der Praxis müssen für ein solches Bündnis aber immer noch zu oft ideologische und/oder ökonomische Barrieren überwunden werden (siehe oben).

\section{Ausblick}

Der Großteil der Publikationen zu «Verhaltenssüchten» ist zu theorielastig. «Die Aufgabe der Wissenschaft besteht aber nicht darin, vollkommene theoretische Schlösser zu errichten, sondern effiziente Interventionstechniken zur Erreichung konkreter Ziele zur Verfügung zu stellen» (frei nach Gregory Bateson). Dazu wäre im Bereich der Verhaltensexzesse seit Jahrzehnten eine patientenbezogene Forschung nötig, um endlich die Inhalte von und die Indikation für VT und/oder Suchtberatung für z.B. spezifische Spieleruntergruppen zu klären!

Zum gegenwärtigen Zeitpunkt hätte die empfohlene pauschale Löschung der Suchtdiagnose angesichts der großen Zahl ambulanter und stationärer Suchteinrichtungen massive strukturelle und personelle Konsequenzen. Sie wäre also auf absehbare Zeit vermutlich nicht umsetzbar. Würde sie umgesetzt, wären die «wirklich» Suchtkranken dann auch weder in der Psychotherapie noch in der Psychiatrie ausreichend versorgt! Eine Extremposition durch eine andere ersetzen zu wollen, behindert nur die Diskussion um patientengerechte Lösungen. Inhaltlich sinnvoll und zügig machbar wäre aber ein Verzicht auf die Suchtdiagnose bei dem Gros der hilfesuchenden Betroffenen mit Verhaltensexzessen.

Im Interesse einer Verbesserung der Patientenversorgung sollten die bestehenden Versorgungsstrukturen also erst einmal adäquat und besser genutzt werden: Das Patientenwohl sollte vor den Machtinteressen von Verbänden und vor dem Wunsch nach Sicherung oder Vermehrung von Arbeitsplätzen für Therapeuten, Forscher oder Berater stehen.

\section{Literatur}

Bilke-Hentsch O, Wölfling K, Batra A (eds): Praxisbuch Verhaltenssucht: Symptomatik, Diagnostik und Therapie bei Kindern, Jugendlichen und Erwachsenen. Stuttgart, Thieme, 2014

Bühringer G, Rumpf H-J: Zukunft der Suchtkrankenversorgung : Plädoyer für einen Paradigma-Wechsel. Sucht 2018;3:125-128.
Hand I: Negative und positive Verstärkung bei pathologischem Glücksspielen: Ihre mögliche Bedeutung für die Theorie und Therapie von Zwangsspektrumsstörungen. Verhaltenstherapie 2004;14:133-144.

Hand I: Strategisch-systemische Aspekte der Verhaltens therapie. Wien, New York, Springer, 2008.

Hand I, Kegat S: Geldflüsse und Aktivitäten seit dem ersten Glücksspielstaatsvertrag: Wie viel, wohin, wofür und mit welchem Ergebnis? Konturen 2013;5:33-39.
KBV (Kassenärztliche Bundesvereinigung): Psychotherapie-Richtlinie. Bundesanzeiger AT,B2 (Strukturreform der ambulanten Psychotherapie), 15.02.2017.

Mann KF (ed): Sonderheft Verhaltenssüchte. Der Nervenarzt 2013;5.

Mann KF (ed): Verhaltenssüchte: Grundlagen, Diagnostik, Therapie, Prävention. Berlin, Heidelberg, Springer, 2014. 